\title{
AVALIAÇÃO DA QUALIDADE DE VIDA EM IDOSOS DE UM CENTRO DE CONVIVÊNCIA
}

\section{EVALUATION OF ELDERLY PEOPLE QUALITY OF LIFE IN A COEXISTENCE CENTER}

\section{EVALUACIÓN DE LA CALIDAD DE VIDA DE LOS ANCIANOS DE UN CENTRO DE CONVIVENCIA}

Aires Garcia dos Santos Junior ${ }^{1}$, Tainara Rocha Casais ${ }^{2}$, Williani Santos Arantes ${ }^{3}$, Fernando Ribeiro dos Santos ${ }^{4}$, Mara Cristina Ribeiro Furlan ${ }^{5}$, Juliana Dias Reis Pessalacia ${ }^{6}$.

\section{RESUMO}

Objetivo: Avaliar a qualidade de vida (QV) em idosos que frequentam um centro de convivência do interior do estado de Mato Grosso do Sul. Método: Foram entrevistados 106 idosos por meio dos instrumentos WHOQoL-BREF e módulo WHOQoL-OLD. Resultados: Apontaram escore total de 62,55 para o WHOQoL-BREF e de 66,06 para o WHOQoL-OLD. O domínio e a faceta com maiores escores foram "Relações sociais" $(67,22)$ e "Funcionamento do sensório" $(70,87)$, respectivamente. Já o domínio e a faceta com os piores escores de satisfação foram o "Ambiente" com 59,96\%, conforme WHOQoL-BREF, e a "Intimidade", com 62,15 de acordo com o WHOQoL-OLD. Conclusão: A avaliação da qualidade de vida em idosos é um indicador importante para a análise da percepção do idoso quanto à sua saúde. Em virtude do amplo contato que o enfermeiro possui com a comunidade, pode-se identificar, por meio da avaliação da QV, as possíveis alterações sensoriais de funcionalidade e das relações sociais que os idosos apresentam e a partir disso propor estratégias e ações de acordo com a real necessidade desta população.

Descritores: Qualidade de vida; Idoso; Envelhecimento; Saúde pública; Centros de Convivência e Lazer.

\section{ABSTRACT}

Objective: To evaluate the quality of life (QoL) in elderly people that attend a community center in the state of Mato Grosso do Sul. Method: We interviewed a hundred six elderly people using the WHOQoL-BREF instruments and the WHOQoL-OLD module. Results: The total score for the WHOQOL-BREF was 62.55 and 66.06 for the WHOQoL-OLD. The domain and the facet with the highest scores were "Social relations" (67.22) and "Sensory functioning" (70.87), respectively. On the other hand, the domain and the factor with the worst satisfaction scores were the "Environment" with 59.96\%, according to WHOQoL-BREF, and the "Intimacy", with 62.15, according to WHOQoL-OLD. Conclusion: The quality of life evaluation in the elderly people is an important indicator for the analysis of the elderly's perception concerning their own health. Due to the nurses' extensive contact with the community, it is possible to identify, through the QoL evaluation, the possible sensorial functional changes and social rellations that the elderly present and then, to propose strategies and actions according to this population real need.

Descriptors: Quality of life; Elderly people; Aging; Public health; Coexistence Centers and Leisure.

\section{RESUMEN}

Objetivo: Evaluar la calidad de vida (CV) de ancianos que frecuentan un centro de convivencia del interior del estado de Mato Grosso del Sur. Método: Se entrevistó a 106 ancianos a través de los instrumentos WHOQoL-BREF y el módulo WHOQoL-OLD. Resultados: Apuntaron puntuación total de 62,55 para el WHOQoL-BREF y de 66,06 para el WHOQoL-OLD. El dominio y la faceta con mayores puntuaciones fueron "Relaciones sociales" $(67,22)$ y "Funcionamiento del sensorio" $(70,87)$, respectivamente. El dominio y la faceta con los peores puntuaciones de satisfacción fueron el "Ambiente" con 59,96\%, según WHOQoL-BREF y la "Intimidad" con 62,15 de acuerdo con el WHOQoL-OLD. Conclusión: La evaluación de la calidad de vida en ancianos es un indicador importante para el análisis de la percepción del anciano en cuanto a su salud. En virtud del amplio contacto que el enfermero posee con la comunidad, se puede identificar, por medio de la evaluación de la CV, las posibles alteraciones sensoriales de funcionalidad y de las relaciones sociales que los ancianos presentan y, a partir de ello, proponer estrategias y acciones de acuerdo con la real necesidad de esta población.

Descriptores: Calidad de vida; Anciano; Envejecimiento; Salud pública; Centros de Ocio y Convivencia.

${ }^{1}$ Graduado em Enfermagem. Doutor em Saúde e Desenvolvimento da Região Centro-Oeste pela Fundação Universidade Federal de Mato Grosso do Sul. Docente do curso de Enfermagem da UFMS. ${ }^{2}$ Graduada em Enfermagem pela Fundação Universidade Federal de Mato Grosso do Sul. ${ }^{3}$ Graduada em Enfermagem pela Fundação Universidade Federal de Mato Grosso do Sul. ${ }^{4}$ Graduando em Medicina pela Fundação Universidade Federal de Mato Grosso do Sul. ${ }^{5}$ Graduada em Enfermagem. Doutora em Saúde e Desenvolvimento da Região Centro-Oeste pela Fundação Universidade Federal de Mato Grosso do Sul. Docente do curso de Enfermagem da UFMS. ${ }^{6}$ Graduada em Enfermagem. Pós Doutora em Enfermagem em Saúde Coletiva pela Escola de Enfermagem da Universidade de São Paulo. Docente do curso de Enfermagem da Fundação Universidade Federal de Mato Grosso do Sul. de Enfermagem do Centro Oeste Mineiro. 2019;9: e3053. [Access___]; Available in:__. DOI: http://dx.doi.org/10.19175/recom.v9i0.3053 


\section{INTRODUÇÃO}

A transição demográfica brasileira vem ocorrendo de forma crescente e acentuada, relacionada, principalmente, com a queda na taxa de fertilidade e aumento da expectativa de vida, sendo um desafio para as equipes de saúde. Aspecto que reforça a necessidade de compreender a sua qualidade de vida (QV), permitindo um melhor planejamento dos serviços de saúde para com esses idosos ${ }^{1}$.

Entre os serviços que são disponibilizados à pessoa idosa, estão os centros de convivência, que são entendidos como espaços de caráter não asilar, no qual são ofertadas atividades: inclusivas, integrativas, recreativas, laborativas, culturais, práticas de exercícios físicos e de cunho cidadãs $^{2}$. Com a Tipificação Nacional dos Serviços Socioassistenciais, foi possível definir as características de funcionalidades essenciais de cada serviço de proteção básica, especial e o seu público alvo ${ }^{3}$.

A Política Nacional do Idoso já afirma a importância dos órgãos públicos em estimular a implementação dos centros de convivência para idosos, como formas diversificadas de proporcionar atendimento a essa população ${ }^{4}$.

No processo de envelhecimento, é frequente que os idosos tenham que conviver, durante anos, com alguma doença crônica. Esse contexto requer uma mudança dos tratamentos tradicionais focados na cura das doenças, faz-se necessário, então, um enfoque na promoção da qualidade de vida e no bem-estar dessa população ${ }^{5}$. A QV no idoso consiste na sua percepção quanto a aspectos biológicos, psicológicos e sociais, tais como a cultura, os valores, os objetivos, as expectativas e as preocupações em relação à vida ${ }^{6}$.

O Código de Ética dos Profissionais de Enfermagem já contempla, entre os seus princípios fundamentais, que o profissional de enfermagem seja comprometido com a QV dos indivíduos como um todo ${ }^{7}$. Sendo assim, é de suma relevância que os profissionais considerem a complexidade da QV, os aspectos culturais e as especificidades de cada indivíduo ${ }^{6}$.

Frente a esse contexto, questiona-se qual a autopercepção de idosos vinculados a um centro de convivência, situado na região do baixo pantanal em relação a sua QV?

Este estudo, portanto, tem como objetivo avaliar a qualidade de vida de idosos que frequentam um centro de convivência do interior do estado de Mato Grosso do Sul.

\section{MÉTODO}

Trata-se de um estudo descritivo e transversal realizado em um munícipio da região norte do estado de Mato Grosso do Sul. Os dados foram coletados por acadêmicos do curso de graduação em Enfermagem, devidamente capacitados pelo professor coordenador do projeto. A pesquisa teve como critérios de inclusão: idosos com idade $\geq 60$ anos, que frequentaram o centro de convivência no período de outubro a dezembro de 2016, e que, após a leitura e assinatura do Termo de Consentimento Livre e Esclarecido (TCLE), em duas vias, aceitaram participar do estudo.

Este estudo está vinculado ao projeto de pesquisa intitulado: 'Centro de Convivência de Idosos: uma abordagem multidimensional e suas implicações para a saúde', o qual foi aprovado pelo parecer número: 1.703 .454 do Comitê de Ética em Pesquisa da Universidade Federal de Mato Grosso do Sul. Após a aplicação dos critérios de inclusão e exclusão, foram considerados para o estudo 106 idosos. Utilizouse o instrumento Brazil Old Age Schedule (BOAS), questionário multidimensional para caracterização sociodemográfica.

Para realizar a avaliação da QV dos idosos, foram empregadas as versões abreviadas em português do WHOQoL-BREF e do WHOQoL-Old module ${ }^{8-9}$.

O instrumento WHOQoL-BREF é composto por 26 questões, das quais, duas questões referentes à qualidade de vida global (QVG) e 24 divididos em quatro domínios da QV, sendo: Físico, Psicológico, Relações Sociais, Meio Ambiente. As respostas de cada domínio variam entre 1 a 5 pontos, seguindo uma escala Likert, para representar as respostas, de acordo com: intensidade (nada - extremamente), capacidade (nada - completamente), frequência (nunca sempre) e avaliação (muito insatisfeito - muito satisfeito; muito ruim - muito bom) ${ }^{8}$.

Os escores de cada domínio são obtidos mediante a soma dos escores da média ' $n$ ' das questões que compõem cada domínio ${ }^{10}$. A mensuração da QV é equivalente ao escore, ou seja, quanto maior for o escore, melhor será a QV do idoso ${ }^{8}$.

O módulo WHOQoL-OLD consiste em 24 itens da escala de Likert atribuídos a seis facetas: Funcionamento do Sensório (FS), Autonomia (AUT), Atividades Passadas, Presentes e Futuras (PPF), Participação Social (PSO), Morte e Morrer (MEM) e Intimidade (INT) ${ }^{9}$. 
Os escores dessas seis facetas ou os valores dos 24 itens do módulo WHOQOL-OLD podem ser associados para produzir um escore geral ('global') de qualidade de vida dos adultos idosos, classificados como o 'escore total' do módulo WHOQOL-OLD ${ }^{9}$.

Foram utilizadas duas ferramentas a partir do software Microsoft Excel para calcular os escores e analisar as estatísticas descritivas (média, desvio padrão, valor máximo, valor mínimo, coeficiente de variação e amplitude) do WHOQoL-BREF e WHOQoL-OLD, seguindo as normas propostas pelo grupo $\mathrm{WHOQ}^{10-11}$.

\section{RESULTADOS E DISCUSSÃO}

Os resultados das análises foram apresentados em uma escala entre 4 e 20 (escore bruto), de acordo com a Tabela 1 e a Tabela 2; enquanto as médias dos escores foram transformadas numa escala de 0 a 100 (escore transformado) e apresentados em gráficos (Figura 1 e Figura 2).

Foram selecionados, para análise desse estudo após a aplicação dos critérios de inclusão e exclusão, 106 idosos, sendo 64,15\% ( $n=68)$ do sexo feminino, $83,96 \% \quad(n=89)$ aposentados, $86,79 \%(n=92)$ possuem idade entre 60 a 79 anos, $43,40 \%(n=46)$ possuem até quatro anos de estudo e $28,30 \%$ ( $n=30)$ são viúvos.

Na tabela 1, indica-se a qualidade de vida dos idosos, onde os valores atribuídos a cada um dos domínios referentes no instrumento WHOQoL-BREF alcançando no instrumento o máximo de 20 pontos.

Tabela 1 - Estatística descritiva da qualidade de vida da amostra. WHOQoL-BREF, escore bruto. Coxim, MS, Brasil, 2016.

\begin{tabular}{|c|c|c|c|c|c|c|}
\hline Domínio & Média & $\begin{array}{l}\text { Desvio } \\
\text { padrão }\end{array}$ & $\begin{array}{l}\text { Coeficiente } \\
\text { de variação }\end{array}$ & $\begin{array}{c}\text { Valor } \\
\text { mínimo }\end{array}$ & $\begin{array}{c}\text { Valor } \\
\text { máximo }\end{array}$ & Amplitude \\
\hline Físico & 13,82 & 1,99 & 14,40 & 8,57 & 17,71 & 9,14 \\
\hline Psicológico & 14,33 & 1,71 & 11,96 & 9,33 & 18,00 & 8,67 \\
\hline Relações Sociais & 14,75 & 2,25 & 15,24 & 4,00 & 18,67 & 14,67 \\
\hline Meio Ambiente & 13,59 & 1,72 & 12,66 & 9,00 & 17,00 & 8,00 \\
\hline Auto-avaliação da Qualidade de Vida & 14,23 & 3,61 & 25,40 & 4,00 & 20,00 & 16,00 \\
\hline
\end{tabular}

Fonte: Elaboração própria.

Um estudo de revisão integrativa demonstrou que a percepção de idosos sobre o envelhecimento saudável, contempla dimensões biopsicossociais e espirituais. $\mathrm{Na}$ dimensão biológica, encontram-se percepções como a necessidade de adotar hábitos e comportamentos saudáveis. Na psicológica, atitudes como o otimismo e a felicidade. $\mathrm{Na}$ espiritual, a fé e a espiritualidade são destacadas como elementos para 0 envelhecimento saudável. Na dimensão social, as relações sociais com a família, amigos e companheiro também influenciam em um envelhecer saudável ${ }^{12}$.

Foi possível observar, na atual investigação, por meio do WHOQOL-BREF valores elevados para o domínio das Relações Sociais. Esses dados corroboram com o encontrado em outros estudos realizados em grupos de convivência de idosos ${ }^{13-}$ ${ }^{14}$. A literatura sugere diferenças nos escores dos domínios de QV entre pessoas em serviços comunitários e em Instituições de Longa
Permanência para Idosos (ILPIs), apontando maiores escores para o domínio Relações Sociais em serviços comunitários não asilares ${ }^{15}$, tal como encontrado neste estudo.

Os resultados demonstram que o domínio Físico $(13,82)$ e o domínio Meio Ambiente $(13,59)$ foram os que menos contribuíram para a QV dos idosos do estudo, de acordo com as médias encontradas. $\mathrm{O}$ valor máximo foi para o domínio Relações Sociais, em que a média encontrada foi de 14,75 e desvio padrão de 2,25 . É pertinente destacar que a prática de atividade física, aulas de dança, jogos, atividades artesanais, proporcionam melhoria da autonomia e da independência dos idosos, favorecendo a confiança dos mesmos ${ }^{16}$.

$\mathrm{Na}$ Figura 1, observa-se que os domínios avaliados, por meio do instrumento WHOQoLBREF (domínios Físico, Psicológico, Relações Sociais e Meio Ambiente estão com satisfação total de 62,55 e que quanto mais se aproximar a 100 , melhor será a QV dos idosos. 
Figura 1 - Qualidade de vida dos idosos de um centro de convivência de idosos. WHOQoL-BREF, escala transformada (0 a 100). Coxim, MS, Brasil, 2016.

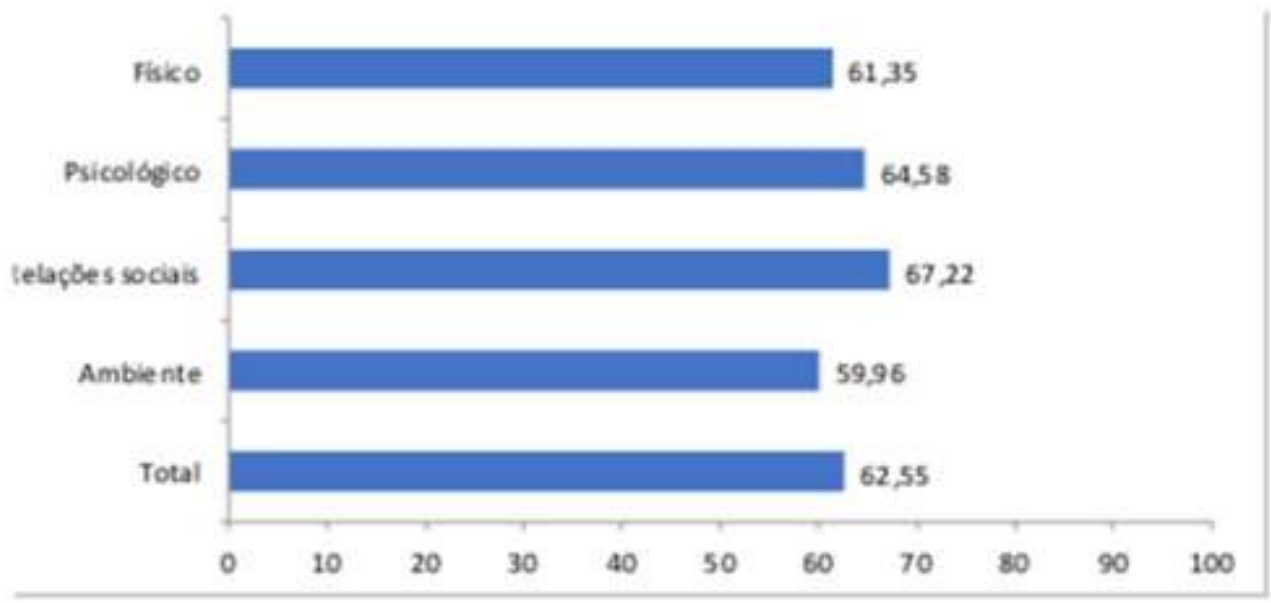

Fonte: Elaboração própria.

Em uma análise específica, o domínio Relações Sociais é o que está com o melhor escore, com 67,22, seguido do domínio Psicológico, com 64,58. O domínio com menor pontuação de satisfação encontrado foi 0 domínio Ambiente, com 59,96.

Um estudo ${ }^{17}$ que também identificou maiores índices no domínio Relações Sociais ressaltou que tais índices possam estar relacionados às maiores oportunidades dos idosos do grupo estudado, de participar de atividades na comunidade.

Verifica-se que o domínio Ambiente foi o que apresentou menor pontuação com média de 13,59 . Esse dado robustece com o encontrado em outro estudo realizado em um centro de referência à pessoa idosa em Belo Horizonte-BH, onde o domínio Ambiente também obteve o pior escore com média de $(14,4)$. Esse domínio está relacionado entre outros fatores com a segurança e a integridade do indivíduo ${ }^{18}$.

Sendo assim, acredita-se que a violência é um aspecto que influencia diretamente na segurança do ambiente em que vive a pessoa idosa, englobando não apenas a agressão física, porém todas as formas de violência contra 0 idoso. Vale destacar que, por diversas vezes, a violência é velada, embutida principalmente em preconceitos $^{18}$.

Nesse contexto, torna-se necessário refletir sobre a formação dos profissionais de saúde, vislumbrando uma assistência holística para o idoso com história de violência. Sabe-se que muitos profissionais não consideram a escuta, o atendimento e a orientação ao idoso nessa condição como suas funções. Grande parte vislumbra apenas os aspectos físicos da violência, com enfoque para a lesão a ela relacionada, deixando de contemplar os aspectos psicossociais relacionados a esse evento ${ }^{19}$.

Além disso, a enfermagem, sendo componente das Equipes de Saúde da Família, possui um papel de suma relevância, ao utilizar como ferramenta de trabalho a visita domiciliar, permitindo ao profissional programar intervenções que favorecem a QV, reduzindo os impactos do ambiente frente ao idoso, além de rastrear situações de vulnerabilidade para a sua saúde, por exemplo, episódios de quedas e sinais indicativos de maus tratos ao idoso ${ }^{18}$.

Outro domínio que teve grande pontuação foi o Psicológico com escore de 64,58. Em estudo realizado com 166 idosos de dois centros de convivência na cidade de São Paulo, identificouse que o domínio Psicológico foi o que apresentou maiores resultados tanto na avaliação do WHOQoL-OLD quanto no WHOQoL-BREF, escores 70,7 e 67,4, respectivamente. Um estudo ${ }^{(20)}$ realizado junto a idosos em condição de vulnerabilidade social identificou correlação entre esse domínio com a fragilidade dos idosos. Também ressaltou a forte influência das atividades presentes, passadas e futuras na satisfação dos idosos e seus objetivos de vida.

Outro estudo $^{21}$ também identificou essa relação entre aspectos emocionais e fragilidade em idosos, ressaltando que a fragilidade, seus componentes e o comprometimento funcional são fatores de risco para a depressão.

Já na tabela 2, mostra-se a média dos valores de cada uma das facetas referentes ao instrumento WHOQOL-OLD, variando de 1 a 5 (sendo que 1 indica insatisfação e 5 satisfação) onde o valor máximo é de 20 pontos. 
Tabela 2 - Estatística descritiva da qualidade de vida da amostra. WHOQoL-OLD, escore bruto. Coxim, MS, Brasil, 2016.

\begin{tabular}{lllllll}
\hline \hline Facetas & Média & $\begin{array}{l}\text { Desvio } \\
\text { padrão }\end{array}$ & $\begin{array}{l}\text { Coeficiente } \\
\text { de variação }\end{array}$ & $\begin{array}{l}\text { Valor } \\
\text { mínimo }\end{array}$ & $\begin{array}{l}\text { Valor } \\
\text { máximo }\end{array}$ & Amplitude \\
\hline $\begin{array}{l}\text { Funcionamento do sensório } \\
\text { Autonomia }\end{array}$ & 15,34 & 3,17 & 20,67 & 8,00 & 20,00 & 12,00 \\
$\begin{array}{l}\text { Atividades passadas, } \\
\text { e futuras }\end{array}$ & 13,15 & 2,62 & 19,91 & 6,00 & 19,00 & 13,00 \\
$\begin{array}{l}\text { Participação social } \\
\text { Morte e montes }\end{array}$ & 14,64 & 1,91 & 13,03 & 9,00 & 19,00 & 10,00 \\
Intimidade & 15,05 & 1,75 & 11,62 & 10,00 & 19,00 & 9,00 \\
\hline \hline
\end{tabular}

Fonte: Elaboração própria.

Nota-se que as facetas AUT e INT obtiveram as médias 13,15 e 13,94 respectivamente, apresentando o pior desempenho em relação aos demais; enquanto as facetas, FS e MEM, obtiveram as maiores médias, respectivamente, 15,34 e 15,29.

Em relação à capacidade funcional dos idosos entrevistados, é possível observar que a faceta AUT foi o que obteve a menor pontuação, por meio da aplicação do WHOQoL-OLD, neste estudo, com 57,19. Em pesquisa realizada em centro de referência à saúde do idoso, na cidade de Campinas, também teve como pior resultado a faceta AUT 47,9 , os autores dessa pesquisa apontam que diversos aspectos podem estar relacionados com a menor pontuação: envolvendo, desde os aspectos econômicos, transporte e até mesmo a quantidade de atividades que são ofertadas pelas instituições ${ }^{22}$. É importante destacar que, no centro de convivência onde foi realizado o presente estudo, são ofertados transporte e refeição aos idosos apenas duas vezes por semana.

Quanto a faceta INT obteve-se escore de $62,15 \%$, o segundo menor resultado na avaliação do WHOQoL-OLD. Esse dado pode estar relacionado com os arranjos familiares, se o idoso vive sozinho e ao seu estado civil, por exemplo, a viuvez e aos divórcios ${ }^{22}$. Contudo, é importante destacar que, mesmo com as eventuais dificuldades que possam surgir frente à fase de envelhecimento, a sexualidade ainda é mantida na vida dos idosos, não se limitando apenas ao ato sexual em si, porém envolvendo desde união, carinho e afeto ${ }^{23}$.

Observa-se, na figura 2, os escores modificados dos tópicos do WHOQoL-OLD, de modo geral, que a qualidade de vida dos idosos foi avaliada de forma regular, com média de 66,06 .

Figura 2 - Qualidade de vida dos idosos de um centro de convivência. WHOQoL-OLD, Escore transformado (0 a 100). Coxim, MS, Brasil, 2016.

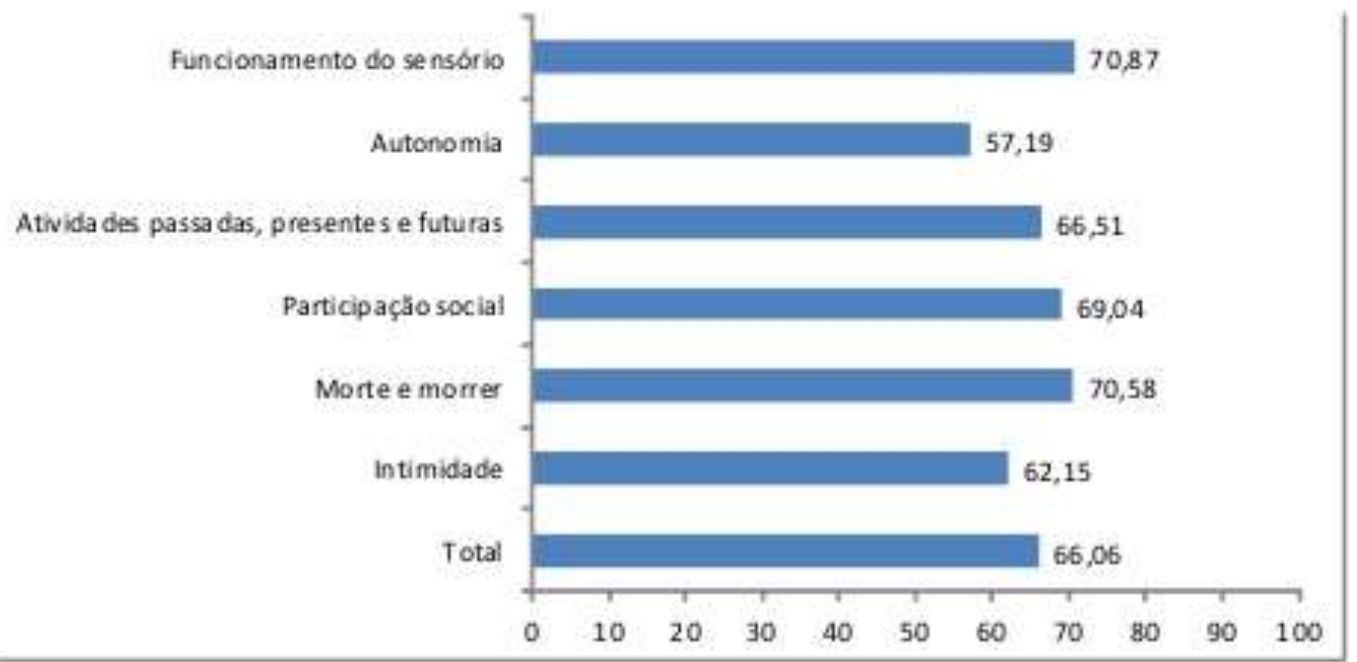

Fonte: Elaboração própria.

Em uma análise específica, a faceta FS com o maior escore $(70,87)$, seguido de MEM $(70,58)$.
Por outro lado, a menor pontuação encontrada foi a faceta AUT $(57,19)$. 
O FS foi a que mais contribuiu para a qualidade de vida, segundo os idosos entrevistados, com média de 15,34 para o WHOQoL-OLD. Em pesquisa realizada em um centro de convivência em Porto Alegre, também se obteve o melhor resultado para a QV com a faceta do FS, tendo escore de 14,8 , os autores reforçam a importância da capacidade funcional, a qual sofre impactos de diversos fatores, desde a saúde física, mental, a independência e a autonomia para as atividades da vida diária ${ }^{14}$. Em estudo realizado com 71 idosos, na cidade de Sorocaba-SP também se obteve o FS como a faceta que mais contribui para a QV dos idosos entrevistados ${ }^{16}$.

No que tange à faceta MEM, observa-se um escore elevado $(70,58)$. Outro estudo ${ }^{20}$ realizado junto a idosos em contexto de vulnerabilidade social também obteve escores elevados nesse quesito. Sabe-se que o domínio morte e morrer demonstra a capacidade de enfrentamento em relação à morte, sendo que o medo do sofrimento no processo de morrer ocasiona maior preocupação do que o evento em si. Nesse contexto, é fundamental abordar os cuidados paliativos na capacitação e formação dos recursos humanos na área da saúde. É importante mencionar ainda, a existência de uma relação positiva entre o avançar da idade e a QV, indicando que quanto mais o idoso ganha a maturidade dos anos, eles tendem a aceitar melhor que o processo de envelhecer é inevitável ${ }^{18}$.

Em relação à faceta PPF que se refere à satisfação com a realização na vida, reconhecimento, a expectativa com o futuro, e metas atingidas no decorrer das experiências ${ }^{16}$, a presente investigação obteve o terceiro pior fator apontado pelos idosos para contribuir com a QV, com média de 14,64. Na literatura ${ }^{14}$, observa-se que essa faceta foi a que menos contribuiu para a QV pelos idosos entrevistados com média de 12,8 . Contudo, cabe enfatizar que nos grupos de idosos podem ser planejados projetos futuros e estimular os mesmos a realizá-los, por meio da concretização de desejos e fortalecendo o sentido na sua existência ${ }^{14}$.

Já em um estudo comparativo entre idosos que frequentam um centro de convivência e idosos residentes em uma ILPI apontam que as facetas: PSO, PPF e AUT apresentaram valores maiores em idosos que frequentam um centro de convivência, visto que, os idosos da ILPI demonstram-se insatisfeitos com sua $\mathrm{QV}^{24}$. Vale destacar que alguns elementos como a escuta qualificada e o desenvolvimento de oficina impulsionam para a comunicação e autonomia dos idosos ${ }^{6}$.

O bem-estar e a QV estão relacionados ao aspecto de um envelhecimento ativo, que irá depender das características econômicas e sociais em que esses idosos estão inseridos ${ }^{24}$. Os grupos de convivência tornam-se locais privilegiados para a socialização de experiências, tornando-se um mecanismo de promoção de saúde e QV, por meio do compartilhamento de informações necessárias ${ }^{25}$.

\section{CONCLUSÃO}

É possível concluir, por meio dos resultados obtidos, que os idosos que frequentaram o centro de convivência apontaram satisfação total com escore de 62,55 para o WHOQoL-BREF e de 66,06 para o WHOQoL-OLD, sendo que o domínio e faceta que mais contribuíram foram 0 de Relações Sociais com 67,22 e do FS com 70,87, segundo o WHOQoL-BREF e O WHOQoL-OLD, respectivamente. Já o domínio e a faceta com piores escores de satisfação foram o Ambiente com 59,96 e a INT com 62,15, considerando o WHOQoL-BREF e O WHOQoLOLD, respectivamente.

A avaliação da QV é um indicador importante da percepção da pessoa idosa em relação a sua situação de saúde, vinculo afetivo, perspectivas, medos e o modo de observar a realidade na qual está inserida. Acredita-se que os achados desse estudo sirvam para nortear o planejamento de ações à população idosa considerando suas reais necessidades.

Frente a esse contexto, a equipe de enfermagem possui um papel fundamental na identificação de agravos e necessidade de suporte, seja de saúde, social ou familiar nos mais diversos contextos. Em virtude do amplo contato que o enfermeiro possui com a comunidade, pode-se identificar, por meio da avaliação da QV, as possíveis alterações sensoriais, de funcionalidade e das relações sociais que os idosos apresentam e propor estratégias e ações de acordo com a real necessidade dessa população. $O$ estudo apresenta como limitação, o fato de ter sido realizado em apenas um centro de convivência de idosos e não ter comparado idosos usuários e não usuários do local. Entretanto, é pertinente apontar que esse estudo faz parte de um grupo limitado de pesquisas disponíveis na literatura, que tiveram por 
finalidade avaliar a QV de idosos em uma região peri-pantaneira.

\section{REFERÊNCIAS}

1 - Bovolenta TM, Felicio AC. How do demographic transitions and public health policies affect patients with Parkinson's disease in Brazil? Clin Interv Aging. 2017;12:197-205. DOI: 10.2147/CIA.S123029

2 - Brasil. Decreto no 1.948, de 3 de julho de 1996. Regulamenta a Lei $n=8.842$, de 4 de janeiro de 1994, que dispõe sobre a Política Nacional do Idoso, e dá outras providências. Diário Oficial da União 1996; 1:12277.

3 - Brasil. Ministério do Desenvolvimento Social e Combate à Fome. Tipificação Nacional de Serviços Socioassistenciais. Brasília: MDS; 2014.

4 - Brasil. Lei no 8.842, de 5 de janeiro de 1994. Dispõe sobre Política Nacional do Idoso e cria o Conselho Nacional do Idoso. Diário Oficial da União 1994;1:77.

5 - Arai H, Ouchi Y, Toba K, Endo T, Shimokado K, Tsubota $\mathrm{K}$ et al. Japan as the front-runner of super-aged societies: Perspectives from medicine and medical care in Japan. Geriatr Gerontol Int. 2015 15(6): 673-87. DOI: $10.1111 /$ ggi.12450

6 - Bassler TC, Santos FR, Santos Junior AG, Furlan MCR, Maia CR. Quality of life evaluation of the elderly living in a for long stay care institution. Rev Enferm UFPE on Line. 2017;11(1):10-7. DOI: 10.5205/reuol.9978-88449-6-1101201702

7 - Conselho Federal de Enfermagem (COFEN). Resolução no 564, de 6 de novembro de 2017. Aprova o novo Código de Ética dos Profissionais de Enfermagem. Diário Oficial da União 2017; 1:157.

8 - Fleck MPA, Louzada $S$, Xavier $M$, Chachamovich E, Vieira G, Santos L et al. Aplicação da versão em português do instrumento de avaliação da qualidade de vida da OMS "WHOQOL-BREF". Rev Saúde Pública 2000;34(2):178-83. DOI: 10.1590/S003489102000000200012

9 - Fleck MP, Chachamovich E, Trentini C. Development and validation of the Portuguese version of the WHOQOL-OLD module. Rev Saúde Pública 2006;40(5):785-91. DOI: 10.1590/S003489102006000600007

10 - Pedroso B, Pilatti LA, Gutierrez GL, Picinin CT. Cálculo dos escores e estatística descritiva do WHOQOL-bref através do Microsoft Excel. RBQV 2010;2(1):31-6. DOI: $\quad 10.3895 / \mathrm{S} 2175-$ $\underline{08582010000100004}$
11 - Pedroso B, Pilatti LA, Gutierrez GL. Cálculo dos escores e estatística descritiva do WHOQOLOLD pelo Microsoft Excel. Geriatr Gerontol. 2010 [citado em 20 jan 2018]; 4(4):214-19. Disponível em: https://sbgg.org.br/publicacoes-cientificas/ revista-geriatria-gerontologia

12 - Tavares RE, Jesus MCP, Machado DR, Braga VAS, Tocantins FR, Merighi MAB. Envelhecimento saudável na perspectiva de idosos: Uma revisão integrativa. Rev Bras Geriatr Gerontol. 2017; 20(6):878-89. DOI: 10.1590/1981$\underline{22562017020.170091}$

13 - Oliveira NS, Pires RML, Lago EC, Batista MRFF, Almeida CAPL. Avaliação da qualidade de vida de idosos que frequentam uma instituição piauiense. Rev Interd. 2015 [citado em 20 jan 2018]; 8(4):47-56. Disponível em: https://revistainterdisciplinar.uninovafapi.edu.br/ index.php/revinter/article/view/688

14 - Serbim AK, Figueiredo AEPL. Qualidade de vida de idosos em um grupo de convivência. Sci Med 2011 [citado em 20 jan 2018]; 21(4):166-72. Disponível em: http://revistaseletronicas.pucrs.br/ojs/index.php/ scientiamedica/article/download/9405/7237 15 - Gamage MWK, Hewage C, Pathirana KD. Effect of cognitive and executive functions on perception of quality of life of cognitively normal elderly people dwelling in residential aged care facilities in Sri Lanka. BMC Geriatr. 2018;18(1):256. DOI: 10.1186/s12877-018-0937-6 16 - Lima BM, Araújo FA, Scattolin FAA. Qualidade de vida e independência funcional de idosos frequentadores do clube do idoso do município de Sorocaba. ABCS Health Sci. 2016; 41(3):168-75. DOI: $10.7322 / a b c s h s . v 41$ i3.907

17 - Tavares DMS, Matias TGC, Ferreira PCS, Pegorari MS, Nascimento JS, Paiva MM. Qualidade de vida e autoestima de idosos na comunidade. Ciênc Saúde Coletiva 2016;21(11): 3557-64. DOI: 10.1590/1413812320152111.03032016

18 - Miranda LCV, Soares SM, Silva PAB. Qualidade de vida e fatores associados em idosos de um Centro de Referência à Pessoa Idosa. Ciênc Saúde Coletiva 2016;21(11):3533-44. DOI: 10.1590/1413-812320152111.21352015

19 - Moreira WC, Carvalho ARB, Lago EC, Amorim FCM, Alencar DC, Almeida CAPL. Formação de estudantes de Enfermagem para atenção integral ao idoso. Rev Bras Geriatr Gerontol. 2018;21(2): 186-93. DOI: $10.1590 / 1981-22562018021.170137$ 20 - Jesus ITM, Diniz MAA, Lanzotti RB, Orlandi FS, Pavarin $\mathrm{SCl}$, Zazzetta MS. Fragilidade e qualidade 
de vida de idosos em contexto de vulnerabilidade social. Texto Contexto - Enferm. 2018;27(4):1-9. DOI: 10.1590/0104-07072018004300016

21 - Lenardt $\mathrm{MH}$, Carneiro NHK, Binotto MA, Willig $\mathrm{MH}$, Lourenço TM, Albino J. Fragilidade e qualidade de vida de idosos usuários da atenção básica de saúde. Rev Bras Enferm. 2016;69(3):

478-83. DOI: 10.1590/0034-7167.2016690309i 22 - Varela FRA, Ciconelli RM, Campolina AG, Soarez PC. Quality of life evaluation of frail elderly in Campinas, São Paulo. Rev Assoc Med Bras. 2015;61(5):423-30. DOI: $\quad \underline{10.1590 / 1806-}$ $\underline{9282.61 .05 .423}$

23 - Marques ADB, Silva RP, Souza SS, Santana RS, Deus SRM, Amorim RF. A vivência da sexualidade de idosos em um centro de convivência. Rev Enferm Cent-Oeste Min. 2015;5(3):1768-83. DOI: 10.19175/recom.v5i3.913

24 - Pereira KCR, Alvarez AM, Traebert JL. Contribuição das condições sociodemográficas para a percepção da qualidade de vida em idosos. Rev Bras Geriatr Gerontol. 2011;14(1):85-95. DOI: 10.1590/S1809-98232011000100010

25 - Ferreira MCG, Tura LFR, Silva RC, Ferreira MA. Representações sociais de idosos sobre qualidade de vida. Rev Bras Enferm. 2017;70(4):806-13. DOI: 10.1590/0034-7167$\underline{\text { 2017-0097 }}$

Nota: Essa pesquisa faz parte do Projeto de Pesquisa "Centro de Convivência de idosos: uma abordagem multidimensional e suas implicações para a saúde".

Received in: 25/07/2018

Approved in: 18/01/2019

Endereço de correspondência:

Aires Garcia dos Santos Junior

Avenida Ranulpho Marques Leal, 3484

CEP: 79613000 - Três Lagoas/MS - Brasil

E-mail: airesjr @hotmail.com 